\title{
Anomalous coronary arteries: What we know and what we do not know
}

\author{
Steven Port, $M D^{\mathrm{a}}$ \\ a Aurora Cardiovascular Services, Milwaukee, WI \\ Received Dec 8, 2015; accepted Dec 9, 2015 \\ doi: $10.1007 /$ s12350-015-0384-0
}

\section{See related article, pp. 226-234}

Clinically recognized coronary anomalies in adults are uncommon. Contemporary imaging studies have demonstrated a prevalence of $1.3 \%$ in the largest direct angiographic series $(n=126,595)$, from 1960 to 1988 at the Cleveland Clinic, ${ }^{1} 2.33 \%$ in a recent consecutive CT coronary angiographic series of 2572 patients from Greece, ${ }^{2}$ and $0.3 \%$ in 59,844 cardiac magnetic resonance scans from England. ${ }^{3}$ Furthermore, not all of the anomalies identified in those studies were of clinical relevance to the patients. Some, such as a high takeoff of a coronary (at or above the sinotubular junction) without an intramural course, an anomalous circumflex coronary originating from the right coronary sinus and coursing posterior to the aorta and separate origins of the circumflex and left anterior descending (LAD) coronary arteries from the left sinus of Valsalva, are typically of no clinical consequence to the patient, although they may be of technical interest to an angiographer. In the aforementioned CT series, those typically benign anomalies represented 41 of the 60 total cases identified.

The classification of coronary anomalies that was used to generate the data in these studies does not include an intramyocardial course of a coronary which may be more common than all of the coronary anomalies. The clinical significance of myocardial bridging remains controversial.

That said, the anomalies that are of clinical importance to a patient include those with anomalous aortic

Reprint requests: Steven Port, MD, Aurora Cardiovascular Services, Milwaukee, WI, USA; sport@wi.rr.com

J Nucl Cardiol 2017;24:235-8.

1071-3581/\$34.00

Copyright (C) 2016 American Society of Nuclear Cardiology. origin of the coronary artery from the opposite sinus of Valsalva (variably abbreviated as AAOCA, ACAOS) or from the pulmonary artery. The term "malignant" has been applied to the subgroup of patients in whom the coronary with an ACAOS has an interarterial course, i.e., coursing between the aorta and the pulmonary artery. As mentioned above, an anomalous circumflex coronary arising from the right coronary sinus of Valsalva virtually invariably takes a posterior course behind the aorta. Hence, the anomalies of particular clinical interest include origin of the left main coronary or the left anterior descending coronary artery (LAD) from the right sinus of Valsalva with an interarterial course and the origin of the right coronary artery (RCA) from the left sinus of Valsalva with an interarterial course. Subsets of the "malignant" anomalies may be more or less dangerous and authors have attempted to ascribe varying risk based on morphological characteristics of the anomalous artery. The acuteness of the angle of takeoff of the anomalous artery, the frequently seen slit-like appearance of the ostium and very proximal part of the anomalous artery (especially of the RCA), and an intramural course of the proximal portion of the anomalous vessel are the most commonly addressed descriptors. In a South Korean study, ${ }^{4}$ patients with anomalous origin of the RCA from the left sinus were divided into "high" and "low" interarterial courses, the former with the ostium of the RCA above the level of the pulmonary valve ("true" interarterial) and the latter with the origin below the pulmonary valve ("right ventricular outflow tract course'"). Subsequent outcome was different between the two subgroups with the high interarterial subgroup experiencing more symptoms and adverse events than the low interarterial group. Intravascular ultrasound data from symptomatic adults with ACAOS involving the left main coronary have shown intussusception of the intramural portion of the anomalous coronary and have suggested that the amount of lateral compression of the intramural portion of the vessel, which can be aggravated with maneuvers that 
increase stroke volume, may be a mechanism of ischemia in that setting. ${ }^{5}$ Why such patients may initially present in the 6th and 7th decade of life remains unclear. One postulate relates to increasing aortic size and consequent compression of the intramurally located anomalous vessel that may accompany age or progressive hypertension.

To get a sense of the clinical impact of the ACAOS, we can look at two population-based reviews. The largest was a study of 6.3 million U.S. Army recruits in which Eckart and associates reviewed the medical records and autopsy reports from 277 non-traumatic fatal events. ${ }^{6}$ Of the 277 deaths, the leading cause of the 64 cardiac deaths was an anomalous coronary artery (21 of $64,34 \%$ ). Furthermore, only an anomalous origin of the left coronary artery from the right sinus of Valsalva (left ACAOS) with an interarterial course was associated with cardiac death. In a series of sudden death in young competitive athletes, ACAOS was the second leading cause of sudden cardiac death, the first being hypertrophic cardiomyopathy. ${ }^{7}$ Of the 27 deaths detailed in the study, 23 were due to a left main ACAOS and 4 due to a right ACAOS. Clearly, these epidemiological reports suggest that the left ACAOS is responsible for far more sudden cardiac deaths than is the right ACAOS.

\section{PREVALENCE OF RIGHT AND LEFT ACAOS}

Left ACAOS was less common $(0.2 \%)$ than was Right ACAOS $(0.35 \%)$ in a CTA study of 2572 adults, aged $29-80 .^{2}$ That distribution was slightly different, perhaps due to either or both the sample size or age (1293), in a Polish CTA study of 8522 subjects, aged 12-93, in which both a Left main/LAD ACAOS and Right ACAOS were found in $0.23 \%$ of the population. In that study, 24 of the 40 subjects $(60 \%)$ with an ACAOS showed an interarterial course. ${ }^{8}$ The largest contemporary CTA study of Right ACAOS comes from South Korea and included 22,925 consecutive patients collected over $5 \frac{1}{2}$ years. The age range was 20-80 yrs. Only those with a Right ACAOS were reported. There were $124(0.5 \%)$ such patients, higher than the prevalence in either the Greek or Polish studies. The most recently reported CTA study included 1519 patients studied at a US military installation over 6 years, from which 22 patients with ACAOS were identified. The CTA was performed for chest pain in $73 \%$ of the group. The average age was 54 yrs. Right ACAOS dominated with $14 / 22$ patients. Of the 22 , there were 12 (55\%) with an interarterial course, all right ACAOS. ${ }^{9}$ Overall, the data suggest that a Right ACAOS occurs with equal or greater frequency but is less frequently associated with a fatal outcome.

\section{SYMPTOMS AND MACE DURING FOLLOW-UP}

Subjects with ACAOS may present without symptoms, their anomalies detected coincidentally or may present with chest pain, palpitations, arrhythmia including atrial fibrillation and ventricular dysrhythmia, syncope, acute coronary syndromes, and sudden cardiac death. The latter occurs most often in younger subjects and is more likely to occur during or soon after vigorous exercise. Retrospective clinical and necropsy studies have estimated the rate of MACE quite variably which is understandable given the selection bias. Few longitudinal studies of subjects with ACAOS are available to generate realistic rates of acute coronary syndromes or sudden cardiac death in subjects who are asymptomatic or newly symptomatic. Contemporary imaging studies of adults that have large enough numbers of subjects have shed some light on the outcomes of individuals with ACAOS. Opolski et al reported a mean follow-up of 15 months for 72 patients with ACAOS. ${ }^{8}$ There were no deaths. Two patients underwent surgery, one for an acute coronary syndrome with no CAD and a right ACAOS and the other for typical angina and a left ACAOS without CAD. Ripley et al reported a median follow-up of 4.6 yrs. for 116 patients with ACAOS. ${ }^{3}$ There were 5 deaths, 4 from myocardial infarction, all of which were attributable to $\mathrm{CAD}$ and 1 from $\mathrm{CHF}$ and CAD. In total, there were 24 myocardial infarctions in the territory of the anomalous coronary artery and all infarctions were related to atherosclerotic plaque. There were no cases of sudden cardiac death due to an ACAOS in the absence of CAD. In a smaller study of symptomatic patients, Clark et al reported on 25-month follow-up of 22 patients with ACAOS. ${ }^{9}$ There were no deaths and one patient with a right ACAOS underwent revascularization for multivessel CAD.

It would appear that sudden cardiac death may be rare in adult subjects with ACAOS and ischemic events in adults are more likely to be related to coronary artery disease than to an ACOAS without CAD.

\section{ROLE OF PROVOCATIVE TESTING}

Sudden cardiac death has been reported in young competitive athletes and in young military recruits with ACAOS and has been documented to occur more frequently either during or soon after vigorous physical exercise. ${ }^{6,7}$ It would, therefore, seem intuitive that traditional exercise stress testing would be a method to identify those subjects with ACAOS who are at increased risk for an adverse cardiac event. However, proof of that concept actually does not exist. While there are individual reports of abnormal exercise myocardial perfusion imaging (MPI) in symptomatic patients with 
ACAOS, no large series exists and no large series exists in asymptomatic subjects with ACAOS. Furthermore, there are case reports of symptomatic individuals such as the 24-year-old man who collapsed while teaching in a classroom and was successfully resuscitated from ventricular fibrillation and proved to have a right interarterial ACAOS as his only pathologic finding. A subsequent exercise MPI was reportedly negative for ischemia. ${ }^{10}$ In the review of sudden cardiac death in 27 young athletes, Basso et al reported that 6 of the 27 subjects with symptoms prior to sudden death had undergone maximum exercise electrocardiography and all 6 were normal. ${ }^{7}$ Conversely, Angelini et al reported that exercise myocardial perfusion imaging was abnormal in 2/3 patients with a symptomatic left ACAOS, the third being too unstable for testing. ${ }^{5}$ The same group has suggested an alternative provocative test involving a rapid saline infusion and dobutamine administration with or without atropine in an attempt to simulate the increased compression of an expanded aorta and pulmonary artery on the intramural ACAOS. There are no large series of patients who have been studied with exercise echocardiography, exercise or vasodilator MPI, or dobutamine MPI or dobutamine echo.

\section{ROLE OF HYBRID IMAGING}

In this issue of the Journal, Gräni et al present their approach to "hybrid" myocardial perfusion and coronary CT angiographic imaging in 46 retrospectively identified patients with ACAOS. ${ }^{11}$ The term "hybrid", is placed in quotations because the patients initially underwent coronary CTA and then, after detection of the ACAOS, had SPECT MPI done. The datasets from the two acquisitions were then fused using commercial software. Regardless of how the datasets are acquired, i.e., fused from separate acquisitions on different machines or from the same hybrid SPECT/CT device, the application of the technology to this patient population may provide important information to guide the clinician faced with a management decision in a patient with an ACAOS. As reviewed above, the outcome of adult subjects with ACAOS may be more often related to coronary artery disease than to the anomalous artery itself. Hybrid SPECT/CTA imaging has the potential to display inducible myocardial ischemia and the relationship of that ischemia to either a nonatherosclerotic ACAOS, an ACAOS complicated by significant $\mathrm{CAD}$, or to $\mathrm{CAD}$ in the normally situated coronaries. In fact, the study by Gräni supports other studies of adult patients with ACAOS in whom the presence of myocardial infarction or stress-induced ischemia was more often related to co-existing CAD than to the coronary anomaly itself. In the current study, 5 patients had ischemia due to CAD in a non-anomalous vessel and 2 patients had ischemia due to CAD in an anomalous vessel. There were 7 patients with a SPECT infarct that were related to CAD in a non-anomalous vessel and 2 patients with infarcts related to CAD in an anomalous vessel. Only 1 infarct could not be precisely assigned to a specific vessel. No patient had an infarct or inducible ischemia in an anomalous vessel free of CAD. In this study, it does not appear that the combination of an ACAOS and CAD in the anomalous vessel is as, or more "'malignant" than CAD in a non-anomalous vessel. Only larger numbers and longer follow-up will answer that question.

\section{TYPE OF STRESS TESTING}

In the current study, 20/46 patients with ACAOS had retroaortic or pre-pulmonic courses and would not be expected to have inducible ischemia in the absence of CAD. There were 26 patients with the so-called "malignant,' i.e., interarterial anatomy. Of those 26 , only 6 subjects had an abnormality on stress MPI. Is it possible that, as suggested by others, the conventional approaches to provocative testing in subjects with ACAOS may be inadequate? Could the absence of ischemia in the $77 \%$ of patients in the current study with a "malignant" ACAOS be due to inadequate stress protocols? The protocols used in this study were quite varied, including bicycle exercise $(30 \%)$, adenosine $(50 \%)$, adenosine plus exercise $(9 \%)$, and dobutamine $(11 \%)$. In young athletes and military recruits who died with an ACAOS, the event typically occurred during or soon after vigorous physical exercise. Are any of the aforementioned stress protocols equivalent to vigorous physical exercise? Do they have to be? Of the 20/26 subjects in the current study with an interarterial ACAOS and a negative MPI study, 17 were free of CAD (Personal communication from the author). Are such patients safe from future non-atherosclerotic MACE? Do their already long-term survivals and negative stress tests indicate that their malignant anatomy is really benign? Do they need any medical therapy? Should we more rigorously study the saline/atropine/dobutamine ("SAD') challenge advocated by one institution? We do not yet have the answers to those questions. Long-term follow-up of those patients is critical to our understanding.

\section{FUTURE DIRECTION}

What we do know is that sudden cardiac death due to an ACAOS appears to largely be a problem for younger subjects, typically those under 30 years of age. We know that sudden cardiac death is most often due to a Left ACAOS. We know that the term "malignant", may be a misnomer in adults since malignant outcomes 
appear to be very rare in middle aged subjects with the so-called malignant anatomy. That may be due to the earlier demise of subjects with truly malignant anatomy or due to the fact that extreme exertion (malignant behavior) is far more common in younger aged subjects. Longitudinal follow-up of older adult subjects identified in the modern era of detection remains relatively short. We know that MACE in middle aged subjects is more often related to co-existing CAD than to the ACAOS itself, although there is some evidence that atherosclerosis is more common in anomalous vessels (not so in the current study). If that were the case, then then the "malignancy" of the interarterial ACAOS in adults may be partly related to a higher propensity to developing significant CAD. We know that there are anatomic markers of risk in ACAOS but how those markers interact with the results of provocative testing is as yet unclear. We know that the widespread application of coronary CTA and cardiac MRA will provide us with the largest population of symptomatic and asymptomatic subjects with ACAOS ever identified and longitudinal studies in those subjects will hopefully expand our knowledge of the approach to such patients. The recent proliferation of single-site studies is of great interest but unlikely to answer the outstanding questions. Multicenter studies or registries will be important. We are aware of true-positive and false-negative stress results in the ACAOS population but how best to challenge the coronary circulation in such patients remains ripe for a rigorous investigation. At this point, "hybrid" SPECT/ CTA applied to adults, as demonstrated in the current study, may represent a valuable tool to begin teasing out the relative contributions of an ACAOS and co-existing $\mathrm{CAD}$ to symptoms and future adverse events.

\section{Disclosure}

None.

\section{References}

1. Yamanak O, Hobbs RE. Coronary artery anomalies in 126,595 patients undergoing coronary arteriography. Cathet Cardiovasc Diagn. 1990;21:28-40.

2. Graidis C, Dimitriadis D, Karasavvidis GD, et al. Prevalence and characteristics of coronary artery anomalies in an adult population undergoing multidetector-row computed tomography for the evaluation of coronary artery disease. BMC Cardiovasc Disord. 2015;15:112-20.

3. Ripley DP, Saha A, Teis A, et al. The distribution and prognosis of anomalous coronary arteries identified by cardiovascular magnetic resonance: 15 year experience from two tertiary centres. J Cardiovasc Magn Reson. 2014;16:34-43.

4. Lee H-J, Yoo JH, Hee YK, et al. Anomalous origin of the right coronary artery from the left coronary sinus with an interarterial course: Subtypes and clinical importance. Radiology. 2012;262:101-8.

5. Angelini P, Walmsley RP, Libreros A, Ott DA. Symptomatic anomalous origin of the left coronary artery from the opposite sinus of valsalva: Clinical presentations, diagnosis and surgical repair. Tex Heart Inst J. 2006;33:171-9.

6. Eckart R, Scoville S, Campbell C, et al. Sudden death in young adults: A 25 -year review of autopsies in military recruits. Ann Int Med. 2004;141:829-34.

7. Basso C, Maron B, Corrado D, Thiene G. Clinical profile of congenital coronary artery anomalies with origin from the wrong aortic sinus leading to sudden death in young competitive athletes. J Am Coll Cardiol. 2000;35:1493-501.

8. Opolski MP, Pregowski J, Mariusz K, et al. Prevalence and characteristics of coronary anomalies originating from the opposite sinus of Valsalva in 8,522 patients referred for coronary computed tomography angiography. Am J Cardiol. 2013;111:1361-7.

9. Clark RA, Marler AT, Lin CK. A review of anomalous origination of a coronary artery from an opposite sinus of Valsalva (ACAOS) impact on major adverse cardiovascular events based $n$ coronary computerized tomography angiography: A 6-year single center review. Ther Adv Cardiovasc Dis. 2014;8:237-41.

10. Jo Y, Uranaka Y, Iwaki H, et al. Sudden cardiac arrest associated with anomalous origin of the right coronary artery from the left main coronary artery. Tex Heart Inst J. 2011;38:539-43.

11. Gräni C, Benz D, Schmied C et al. Hybrid CCTA/SPECT myocardial perfusion imaging findings in patients with anomalous origin of coronary arteries from the opposite sinus and suspected concomitant coronary artery disease. J Nucl Cardiol. 